\title{
Two strategies underlying the trade-off of hepatitis $C$ virus proliferation: stay-at-home or leaving-home?
}

\section{Authors:}

Shoya Iwanami ${ }^{1, \dagger}$, Kosaku Kitagawa ${ }^{1, \dagger}$, Yusuke Asai², Hirofumi Ohashi ${ }^{3,4}$, Kazane Nishioka ${ }^{3,4}$, Hisashi Inaba5, Shinji Nakaoka ${ }^{6,7}$, Takaji Wakita ${ }^{3}$, Odo Diekmann ${ }^{8}$,

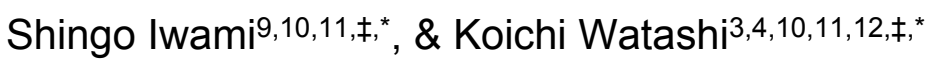

\section{Affiliations:}

${ }^{1}$ Graduate School of Systems Life Sciences, Kyushu University, Fukuoka 819-0395, Japan. ${ }^{2}$ Graduate School of Medicine, Hokkaido University, Hokkaido 060-8638, Japan. ${ }^{3}$ Department of Virology II, National Institute of Infectious Diseases, Tokyo 162-8640, Japan. ${ }^{4}$ Department of Applied Biological Science, Tokyo University of Science, Noda 278-8510, Japan. ${ }^{5}$ Graduate School of Mathematical Sciences, The University of Tokyo, Tokyo 1538914, Japan. ${ }^{6}$ Faculty of Advanced Life Science, Hokkaido University, Sapporo 060-0810, Japan. ${ }^{7}$ PRESTO, Japan Science and Technology Agency, Saitama 332-0012, Japan. ${ }^{8}$ Mathematisch Institute, Universiteit Utrecht, 3508 TA Utrecht, The Netherlands. ${ }^{9}$ Department of Biology, Faculty of Sciences, Kyushu University, Fukuoka 819-0395, Japan. ${ }^{10 M I R A I, ~ J S T, ~ S a i t a m a ~}$ 332-0012, Japan. ${ }^{11}$ CREST, JST, Saitama 332-0012, Japan. ${ }^{12}$ Institute for Frontier Life and Medical Sciences, Kyoto University, Kyoto 606-8507, Japan.

$\dagger, \ddagger$ These authors contributed equally to this study.

* Correspondence and requests for materials should be addressed to S.I. (email: siwami@kyushu-u.org) or K.W. (email: kwatashi@nih.go.jp). 


\section{Abstract (244/250)}

Viruses proliferate through both genome replication inside infected cells and transmission to new target cells or to new hosts. Each viral genome molecule in infected cells is used either for amplifying the intracellular genome as a template ("stay-at-home strategy") or for packaging into progeny virions to be released extracellularly ("leaving-home strategy"). The balance between these strategies is important for both initial growth and transmission of viruses. In this study, we used hepatitis $\mathrm{C}$ virus $(\mathrm{HCV})$ as a model system to study the functions of viral genomic RNA in both RNA replication in cells and in progeny virus assembly and release. Using viral infection assays combined with mathematical modelling, we characterized the dynamics of two different HCV strains (JFH-1, a clinical isolate, and Jc1-n, a laboratory strain), which have different viral assembly and release characteristics. We found that $1.27 \%$ and $3.28 \%$ of JFH-1 and Jc1-n intracellular viral RNAs, respectively, are used for producing and releasing progeny virions. Analysis of the Malthusian parameter of the HCV genome (i.e., initial growth rate) and the number of de novo infections (i.e., initial transmissibility) suggests that the leaving-home strategy provides a higher level of initial transmission for Jc1-n, while, in contrast, the stay-at-home strategy provides a higher initial growth rate for JFH-1. Thus, theoretical-experimental analysis of viral dynamics enables us to better understand the proliferation strategies of viruses. Ours is the first study to analyze stay-leave trade-offs during the viral life cycle and their significance for viral proliferation.

\section{Keywords:}




\section{Introduction}

Hepatitis $\mathrm{C}$ virus (HCV) is an RNA virus specifically infecting liver cells. HCV produces progeny viruses rapidly, with $\sim 10^{12}$ copies sometimes observed in patients [1]. Following virus entry into target cells, viral genomic RNA produces structural proteins (S) and non-structural proteins (NS) (Fig. 1A). Using the genomic RNA as a template, the viral non-structural proteins amplify HCV RNA ("RNA replication"). Genomic RNA can also be assembled with viral structural proteins into progeny virions to be egressed outside of cells, creating the opportunity for transmission (in this study, we call the process including particle assembly and egress "release"). Thus, a single HCV genomic RNA molecule can be used either for RNA replication or for release, and the balance between these processes governs viral proliferation. The molecular mechanisms underlying each event in the viral life cycle have been extensively investigated [2, 3], yet the replication-release trade-off and its significance for viral proliferation remain poorly understood.

$\mathrm{HCV} \mathrm{JFH}-1$ is a genotype 2a strain isolated by our group from a patient with fulminant hepatitis [4]. JFH-1 has been a standard strain used for experiments to characterize $\mathrm{HCV}$ infection, virus-host interactions, and immune responses against HCV [4]. In addition, Jc1 or J6/JFH (a chimeric strain in which a region of the JFH-1 genome from the core to NS2 was replaced by sequences from another genotype $2 a$ virus, the J6 strain) was developed as a laboratory strain to improve virus production, and used for development of antiviral agents and vaccines, which requires large amounts of virus $[5,6]$. In spite of their high sequence similarity $(97 \%$ identity over the whole genome), these two viruses have different virological characteristics especially in terms of the release process: while JFH-1 particles assemble on lipid droplet membranes, particle assembly of $\mathrm{J6} / \mathrm{JFH}-1$-chimeric lab strains is associated with endoplasmic reticulum-derived membranes $[2,3]$. Thus, these two related strains are a useful a reference set to compare the dynamics of release and RNA replication.

In this study, we used a cell culture model of infection with these two HCV reference strains and measured the time-course of viral production (including HCV RNA inside cells and virions produced outside of the cells), infectivity of progeny HCV, and infected cell numbers. We also developed a multiscale mathematical model to describe intra- and inter-cellular HCV dynamics. This interdisciplinary approach suggests that different strategies exist for viral proliferation: the stay-at-home strategy (JFH-1) and the leaving-home strategy (Jc1-n, a J6/JFH-1-chimeric strain). We 
bioRxiv preprint doi: https://doi.org/10.1101/821710; this version posted October 28, 2019. The copyright holder for this preprint (which was not certified by peer review) is the author/funder, who has granted bioRxiv a license to display the preprint in perpetuity. It is made available under aCC-BY 4.0 International license.

60 discuss the relevance of these strategies for viral proliferation, while referring to [7] for 61 wider evolutionary context. 


\section{Results}

\section{Age-structured multiscale modeling of HCV infection}

64

To describe the intracellular replication dynamics of HCV viral RNA, we used the following mathematical model:

66

$$
\begin{aligned}
& \frac{d R(a)}{d a} \\
& =k R(a)-(\mu+\rho) R(a),
\end{aligned}
$$

where $R(a)$ is the amount of intracellular viral RNA in cells that have been infected

$a$. The intracellular viral RNA replicates at rate $k$, degrades at rate $\mu$, and is released to extracellular space at rate $\rho$ (Fig. 1B). Note that if viruses have small or large $\rho$, then they tend to stay inside or leave the cell, respectively (see later). In our virus experiments (see Methods), the released viruses could infect other target cells. To describe multi-round virus transmission (i.e., de novo infection), we needed to couple intracellular viral replication with a standard mathematical model for intercellular virus infection in cell culture [8, 9] (Fig. 1C). In Supplementary Note 1, we derived the following multiscale ordinary differential equation (ODE) model for $\mathrm{HCV}$ infection from the corresponding age-structured partial differential equation (PDE) model [10, 11]:

$$
\frac{d T(t)}{d t}
$$

79

$$
=g T(t)\left(1-\frac{T(t)+I(t)}{K}\right)-\beta_{\theta} T(t) V_{\theta}(t),
$$

$$
\frac{d I(t)}{d t}
$$

80

$$
=g I(t)\left(1-\frac{T(t)+I(t)}{K}\right)+\beta_{\theta} T(t) V_{\theta}(t)
$$

$$
=\beta_{\theta} T(t) V_{\theta}(t)+(k-\mu-\rho) A(t),
$$

$$
\begin{aligned}
& \frac{d V_{\theta}(t)}{d t} \\
& =f_{\theta} \rho A(t)-r V_{\theta}(t)-c V_{\theta}(t),
\end{aligned}
$$




$$
\begin{aligned}
& \frac{d V(t)}{d t} \\
& =\rho A(t)-c V(t) .
\end{aligned}
$$

(6)

Here, the intercellular variables $T(t)$ and $I(t)$ represent the numbers of uninfected and infected target cells, and $V(t)$ and $V_{\theta}(t)$ represent the total amount of extracellular viral RNA (copies/well) and the extracellular viral infectious titer expressed (ffu/well), respectively. The intracellular variable $A(t)$ represents the total amount of intracellular viral RNA. The parameters $g$ and $K$ represent the growth rate and the carrying capacity of target cells, and $\beta_{\theta}$ and $f_{\theta}$ are the converted infection rate constant and the fraction of infectious virus, respectively. We assumed that progeny viruses were cleared at rate $c$, and that infectious virions lose infectivity at rate $r$. Separately, we directly estimated $g, K, c, \mu+\rho$ and $r$ for both HCV JFH-1 and Jc1-n in Fig. S1. Detailed explanations of Eqs. (2-6) are given in

\section{Supplementary Note 1.}

To assess the variability of kinetic parameters and model predictions, we performed Bayesian estimation for the whole dataset using Markov chain Monte Carlo (MCMC) sampling (see Methods). Simultaneously, we fitted Eqs. (2-6) to the experimentally-determined numbers of uninfected and infected cells, extracellular viral RNA (copies/well) and infectious titer (ffu/well), and intracellular viral RNA (copies/well). These figures were derived from infection experiments using different numbers of plated cells for either HCV JFH-1 or Jc1-n as described previously [8, 9, $12,13]$. The remaining free model parameters (i.e., $\beta_{\theta}, k, \rho, f_{\theta}$ ) along with initial values for variables (i.e., $\left.T(0), I(0), A(0), V_{\theta}(t), V(0)\right)$ were determined. Experimental measurements below the detection limit were excluded in the fitting. The estimated parameters and initial values are listed in Table 1 and Table S1. The typical behavior of the model using these best-fit parameter estimates is shown together with the data in Fig. 2A for HCV JFH-1 (orange) and Jc1-n (green) (see Methods for HCV strains), and indicated that Eqs. (2-6) described the in vitro data very well. The shadowed regions corresponded to $95 \%$ posterior predictive intervals, the solid and dashed lines gave the best-fit solution (mean) for Eqs. (2-6), and the orange circles and green triangles showed the experimental datasets.

Using the estimated parameters shared between the original PDE model in Supplementary Note 1 and the transformed ODE model (i.e., Eqs. (2-6)), we successfully reconstructed age information for intracellular viral RNA in infected cells of infection age $a$, which cannot be obtained through conventional experiments alone 
115 (Fig. 2B). Fig. 2C shows the differences in intracellular JFH-1 and Jc1-n viral RNA

116 levels in cells of infection age $a$. At the beginning of the experiment, intracellular viral

117 RNA increased faster under Jc1-n infection than under JFH-1 infection (shown in

118 green). However, intracellular JFH-1 viral RNA gradually accumulated to higher levels

119 than Jc1-n at later time points after infection (shown in yellow to brown). These data

120 illustrated the different dynamics of these two strains and the impact of these

121 dynamics on intracellular viral RNA production, all resulting from different strategies

122 to transmit the viral genome (see below).

\section{Dynamics of HCV JFH-1 and Jc1-n strain replication}

Our model [Eqs. (2-6)] applied to time-course experimental data allowed us to extract the following kinetic parameters: the distribution of the rate constant for infection, $\beta_{\theta}$, the release rate of intracellular viral RNA, $\rho$, the degradation rate of intracellular viral RNA, $\mu$, the converted fraction of infectious viral RNA, $f_{\theta}$, and the replication rate of intracellular viral RNA, $k$ (Fig. 3 and Table 1). Comparing these parameters for $\mathrm{JFH}-1$ and $\mathrm{Jc} 1-\mathrm{n}$ showed a significant difference between the rate constant for infections, $\beta_{\theta}$, of JFH-1 $\left(1.29 \times 10^{-4}, 95 \% \mathrm{Cl}: 0.81-1.92 \times 10^{-4}\right)$ and Jc1-n $\left(2.21 \times 10^{-4}, 95 \% \mathrm{Cl}: 1.69-2.77 \times 10^{-4}\right)\left(p=1.82 \times 10^{-4}\right.$ by repeated bootstrap $t$-test) (Fig. 3A). In addition, the release rate of intracellular viral RNA, $\rho$, for JFH-1 and Jc1-n were $2.43 \times 10^{-2}\left(95 \% \mathrm{Cl}: 1.87-3.11 \times 10^{-2}\right)$ and $6.25 \times$ $10^{-2}\left(95 \% \mathrm{Cl}: 4.62-8.44 \times 10^{-2}\right)$, respectively $\left(p=2.00 \times 10^{-6}\right.$ by repeated bootstrap t-test) (Fig. 3B). These estimates indicated that Jc1-n infects cells 1.71 times faster and produces progeny viruses from infected cells 2.57 times faster than $\mathrm{JFH}-1$. The estimate was further validated by independent experiments, in which Jc1-n entry and virus production were indeed significantly higher than those of JFH-1

140 (Supplementary Note 2 and Fig. S2). There was also a small but significant difference between the degradation rate, $\mu$, of $\mathrm{JFH}-1(0.78,95 \% \mathrm{Cl}: 0.77-0.78)$ and Jc1-n (0.83, 95\% Cl: $0.80-0.84)$ (Fig. 3C). No significant difference was apparent in the converted fraction of infectious virus, $f_{\theta}$ (Fig. 3D). Because JFH-1 and Jc1-n have identical non-structural regions essential for RNA replication (NS3-NS5B), we estimated the same viral RNA replication rate, $k$, for these two viruses (Fig. 3E). Hence, our parameter estimation captured the characteristics of the two strains well and was able to quantitatively describe viral infection dynamics.

In our multiscale model (Eqs. (2-6)), the accumulation rate of intracellular viral RNA was defined as the difference between the replication rate and the sum of 
150 the degradation rate and the release rate (i.e., $k-\mu-\rho$ ). The distributions of

151 calculated intracellular RNA accumulation rates for JFH-1 $(1.11,95 \% \mathrm{Cl}: 1.04-1.18)$

152 and Jc1-n $(1.02,95 \% \mathrm{Cl}: 0.95-1.09)$ are shown in Fig. $3 \mathbf{F}\left(p=1.58 \times 10^{-3}\right.$ by

153 bootstrap $t$-test) (Table 1). The preferential accumulation of JFH-1 RNA inside cells

154 was consistent with its tendency toward gradual increased levels of intracellular RNA

155 at later time points (Fig. 2C). To further evaluate total viral RNA level considering

156 multi-round virus transmission, the Malthusian parameter, $M$, was used as an

157 indicator of the initial growth rate of intracellular viral RNA for each HCV strain [8, 12,

158 14]. Here, the Malthusian parameter was given by

$$
M=\frac{k-\mu-\rho-r-c+\sqrt{(k-\mu-\rho+r+c)^{2}+4 \beta_{\theta} K f_{\theta} \rho}}{2} .
$$

The Malthusian parameters for JFH-1 and Jc1-n were calculated as 1.11 (95\% Cl: $1.04-1.18)$ and $1.02(95 \% \mathrm{Cl}: 0.95-1.09)$, respectively, and were significantly different from one another ( $p=1.02 \times 10^{-3}$ by bootstrap $t$-test) (Fig. 3G and Table 1). Interestingly, even if Jc1-n had a larger infection rate, $\beta_{\theta}$, and release rate, $\rho$, compared with $\mathrm{JFH}-1$, the initial growth rate of total JFH-1 RNA was higher than that of Jc1-n. This result demonstrated that the capacity to accumulate viral RNA inside cells predominantly determines the initial growth rate rather than release of progeny viruses.

\section{Stay-at-home strategy or leaving-home strategy for "optimizing" HCV} proliferation

We investigated how differences between the two strains, JFH-1 and Jc1-n, might be interpreted in an evolutionary perspective. As mentioned above, we considered two opposing strategies: the "stay-at-home strategy" and the "leaving-home strategy": If viruses have smaller $\rho$, they preferentially stay inside the cell, but if they have larger $\rho$, they leave the cell. To quantitatively characterize these different strategies, we defined the fraction of viral RNA remaining in the cells ( $(k-\mu-\rho) / k)$, released from the cells $(\rho / k)$, and degraded in the cells $(\mu / k)$ within the total intracellular viral RNA produced (Fig. 4A). Using all accepted MCMC parameter estimates from the time-course experimental datasets, we calculated that the

180 fractions of viral RNA remaining were $57.9 \%$ and $53.5 \%$, the fractions of viral RNA 181 degraded were $40.8 \%$ and $43.2 \%$, and the fractions of viral RNA released were $1821.27 \%$ and $3.28 \%$ for JFH-1 and Jc1-n, respectively (Fig. 4B). Notably, Jc1-n used intracellular viral RNA for virus release 2.58 times faster than $\mathrm{JFH}-1$, explaining the 
rapid transmission of Jc1-n (Fig. 2C). These results indicate the preferential "leaving-home" strategy of Jc1-n as compared with JFH-1, which adopts a "stay-at-home" strategy.

To further investigate these two opposing strategies, we addressed the relevance of viral RNA release rates for viral proliferation using in silico analysis. With various values of the release rate of intracellular viral RNA, $\rho$, we calculated the Malthusian parameter for each strain as an indicator of viral fitness (Fig. 4C). Each curve shows Malthusian parameters calculated using 100 parameter sets sampled from MCMC parameter estimates as functions of $\rho$, and each gray vertical line is the corresponding estimated release rate. Interestingly, the smaller release rate, the larger the Malthusian parameter HCV achieves. This is because intracellular viral RNAs can be amplified faster compared with viral RNAs outside of cells that are degraded or enter new cells. This result showed that the JFH-1 strain is more optimized in terms of its Malthusian parameter compared with Jc1-n because of the smaller estimated values of $\rho$. That is, $\mathrm{HCV} \mathrm{JFH-1}$ adopts the stay-at-home strategy for acquiring a higher initial growth rate. evaluate viral transmissibility:

Next, we defined the cumulative number of newly infected cells at time $t$ to

We also calculated the cumulative number of newly infected cells for each strain using the means of the estimated parameters as functions of $\rho$ (Fig. 4D). Each curve showed the calculated cumulative number of infected cells until 2, 4, 6, 8, and 10 days post-infection, and the gray vertical line represented the mean release rate estimated from the infection experiment. The value of the release rate, which maximized the cumulative number of newly infected cells, was between 0.1 and 1 . This is because an intermediate release rate effectively increases extracellular viral

210 RNA for new infection: Lower release rates do not effectively produce new infections

211 while higher release rates decrease intracellular viral RNA levels and thus diminish

212 future new infections. Thus, it appears that Jc1-n is more optimized for producing 213 newly infected cells. This implies that HCV Jc1-n adopts the leaving-home strategy to acquire an advantage in producing newly-infected cells.

Taken together, our theoretical investigation based on viral infection

216 experiments revealed that the JFH-1 strain optimizes its initial growth rate, but the 217 Jc1-n strain optimizes de novo infection. Ours is the first report to quantitatively 
bioRxiv preprint doi: https://doi.org/10.1101/821710; this version posted October 28, 2019. The copyright holder for this preprint (which was not certified by peer review) is the author/funder, who has granted bioRxiv a license to display the preprint in perpetuity. It is made available under aCC-BY 4.0 International license.

218 evaluate these opposing evolutionary strategies and to show their significance for 219 virus proliferation at the intracellular and intercellular levels. 


\section{Discussion}

Through a combined experimental-theoretical approach, we analyzed the dynamics of the HCV life cycle using two related HCV strains, JFH-1 and Jc1-n, employing different particle assembly/release strategies. We quantified the intra- and inter-cellular viral dynamics of these strains by applying an age-structured multiscale model to time-course experimental data from an HCV infection cell culture assay (Fig. 2A and Table 1): As in [10,11], we transformed the multiscale model formulated by PDEs to an identical multiscale ODE model (i.e., Eqs. (2-6)), and estimated parameters shared between the PDE and ODE models. It is technically challenging to obtain experimental measurements with age information, but thanks to the estimated values of these common parameters, we managed to reconstruct age information for intracellular viral RNA (Fig. 2BC). In addition, comparing the calculated Malthusian parameters and the cumulative number of newly infected cells between the two strains (Fig. 3FG), we found that the JFH-1 strain had a higher initial growth rate but that Jc1 produced more de novo infections.

Based on our results, we propose two opposing strategies for viral proliferation: the "stay-at-home strategy" and the "leaving-home strategy." From an evolutionary perspective, JFH-1 adopts a stay-at-home strategy and preferentially uses viral genomic RNA for increasing intracellular replication. In contrast, adopting a leaving-home strategy, Jc1-n uses more viral genomic RNA for producing progeny virions capable of new transmission events to increase the number of infected cells (Fig. 4). Thus, Jc1-n infects cells 1.71 times faster and produces viral RNA from infected cells 2.57 times faster than JFH-1. Our group and others reported that JFH-1 assembled progeny virions on the membranes of hepatic lipid droplets, while J6/JFH-1 chimeric strains mainly used endoplasmic reticulum-derived membranes for particle production [2, 3]. Although the molecular aspects of this difference have been analyzed, its significance for viral proliferation and dynamics is not completely understood. Our results raise the possibility that different subcellular locations for particle assembly impact the rates of particle assembly and release, which in turn determine virus proliferation. Further analysis might shed light on why one HCV strain has to be assembled on the lipid droplet membrane while another assembles in association with the endoplasmic reticulum.

The choice of replication strategy not only determines virus proliferation but also affects the pathogenic features of the virus: JFH-1, which preferentially amplifies intracellular RNA, caused fulminant hepatitis with rapid viral replication and severe 
255 inflammation. By contrast, J6, the original strain encoding the Jc1-n structural region,

256 was isolated from a patient with chronic hepatitis and generally replicates more

257 moderately, with robust spread of infected cells used as a longer term strategy to

258 establish persistent infection. Characterization of the proliferation strategies of viruses

259 is of significant importance when trying to understand their clinical as well as

260 evolutionary properties. 


\section{Methods}

262

\section{Cell culture and HCV infection}

Huh-7.5.1 (kindly provided by Dr. Francis Chisari, The Scripps Research Institute) and Huh7-25 cells were cultured in Dulbecco's Modified Eagle's Medium (Invitrogen) supplemented with $10 \%$ fetal bovine serum (Sigma), 10 units $/ \mathrm{mL}$ penicillin, $10 \mathrm{mg} / \mathrm{mL}$ streptomycin, $0.1 \mathrm{mM}$ non-essential amino acids (Invitrogen), 1 $\mathrm{mM}$ sodium pyruvate, and $10 \mathrm{mM}$ HEPES, $\mathrm{pH} 7.4$, at $37^{\circ} \mathrm{C}$ under a humidified atmosphere containing $5 \% \mathrm{CO}_{2}$. We used $\mathrm{HCV}$ strains $\mathrm{JFH}-1$, a genotype 2 a clinical isolate from a patient with fulminant hepatitis [4], and Jc1-n, a J6/JFH-1 chimeric laboratory strain [13]. JFH-1 and Jc1-n have 96.7\% amino acid identity over the whole genome. HCV inoculum for infection experiments was recovered from the culture supernatants of Huh-7.5.1 cells transfected with the corresponding HCV RNA as described [4]. Huh-7.5.1 cells were inoculated with JFH-1 or Jc1-n for $4 \mathrm{~h}$ and then passaged to seed a new 96 well plate at different cell densities (1000, 2000, or 4000 cells/well). At days $0,1,2,3$, and 4 post-seeding, culture supernatants and cell lysates were recovered to quantify HCV RNA by real time RT-PCR as previously described [13]. The infectivity of HCV in culture supernatants was measured using a focus-forming assay as described [13]. To quantify the number of uninfected and infected cells, cells were fixed and stained with anti-HCV core antibody by immunofluorescence assay as described [13].

\section{Data fitting and parameter estimation}

The parameters $g$ and $K$ were separately estimated (see Supplementary Note 3 ) and fixed at 0.660 and $4.12 \times 10^{4}$, respectively, for the JFH-1 strain, and 0.665 and $3.75 \times 10^{4}$, respectively, for the Jc1-n strain. A statistical model adopted from Bayesian inference assumed that measurement error followed a normal distribution with mean zero and unknown variance (error variance). A distribution of error variance was also inferred using the Gamma distribution as its prior distribution.

The posterior predictive parameter distribution as an output of MCMC computation represented parameter variability. Distributions of model parameters (i.e., $\beta_{\theta}, k, \rho$, $f_{\theta}$ ) and initial values (i.e., $\left.T(0), I(0), A(0), V_{\theta}(t), V(0)\right)$ in Eqs. (2-6) were inferred directly by MCMC computations. Distributions of derived quantities were calculated from the inferred parameter sets (Fig. 3EF for graphical representation). A set of computations for Eqs (2-6) with estimated parameter sets gives a distribution of outputs (the number of cells and the intra- and extra-cellular viral loads) as model 
296 predictions. To investigate variation in model predictions, global sensitivity analyses

297 were performed. The range of possible variation is shown in Fig. $\mathbf{2 A}$ as $95 \%$

298 confidence intervals. Technical details of MCMC computations are summarized

299 below.

300

301 Statistical analysis

302 Package FME [15] in R Statistical Software [16] was used to infer posterior

303 predictive parameter distributions. The delayed rejection and Metropolis method [17]

304 was used as a default computation scheme for FME to perform MCMC computations.

305 MCMC computations for parameter inference were implemented using the

306 pre-defined function modMCMC() in package FME as introduced in Methods.

307 Convergence of Markov chains to a stationary distribution was required to ensure

308 parameter sets were sampled from a posterior distribution. Only the last 90000 of

309100000 chains were used as burn-in. The convergence of the last 90000 chains was

310 manually checked with figures produced by package coda [18], a collection of

311 diagnostic tools for MCMC computation. The 95\% credible interval shown as a

312 shadowed region in each panel of Fig. 2A was produced from 100 randomly chosen

313 inferred parameter sets and corresponding model predictions. We employed a

314 bootstrap $t$-test [19] to quantitatively characterize differences in parameters and

315 derived quantities between HCV JFH-1 and Jc1-n. In total, 100000 parameter sets

316 were sampled with replacement from the posterior predictive distributions to calculate

317 the bootstrap t-statistics. To avoid potential sampling bias, the bootstrap t-test was

318 performed 100 times repeatedly. The averages of the computed $p$-values were used

319 as indicators of differences. 
1. Neumann AU, Lam NP, Dahari H, Gretch DR, Wiley TE, Layden TJ, et al. Hepatitis $C$ viral dynamics in vivo and the antiviral efficacy of interferon-alpha therapy. Science. 1998;282(5386):103-7. Epub 1998/10/02. PubMed PMID: 9756471. 2. Miyanari Y, Atsuzawa K, Usuda N, Watashi K, Hishiki T, Zayas M, et al. The lipid droplet is an important organelle for hepatitis $C$ virus production. Nat Cell Biol. 2007;9(9):1089-97. Epub 2007/08/28. doi: 10.1038/ncb1631. PubMed PMID: 17721513.

3. Boson B, Granio O, Bartenschlager R, Cosset FL. A concerted action of hepatitis $C$ virus $p 7$ and nonstructural protein 2 regulates core localization at the endoplasmic reticulum and virus assembly. PLoS Pathog. 2011;7(7):e1002144. Epub 2011/08/05. doi: 10.1371/journal.ppat.1002144. PubMed PMID: 21814513; PubMed Central PMCID: PMCPMC3141040.

4. Wakita T, Pietschmann T, Kato T, Date T, Miyamoto M, Zhao Z, et al. Production of infectious hepatitis $C$ virus in tissue culture from a cloned viral genome. Nat Med. 2005;11(7):791-6. Epub 2005/06/14. doi: 10.1038/nm1268. PubMed PMID: 15951748; PubMed Central PMCID: PMCPMC2918402.

5. Lindenbach BD, Evans MJ, Syder AJ, Wolk B, Tellinghuisen TL, Liu CC, et al. Complete replication of hepatitis $C$ virus in cell culture. Science. 2005;309(5734):623-6. Epub 2005/06/11. doi: 10.1126/science.1114016. PubMed PMID: 15947137.

6. Pietschmann T, Kaul A, Koutsoudakis G, Shavinskaya A, Kallis S, Steinmann $E$, et al. Construction and characterization of infectious intragenotypic and intergenotypic hepatitis $C$ virus chimeras. Proceedings of the National Academy of Sciences of the United States of America. 2006;103(19):7408-13. Epub 2006/05/03. doi: 10.1073/pnas.0504877103. PubMed PMID: 16651538; PubMed Central PMCID: PMCPMC1455439.

7. Dieckmann U, Metz JA, Sabelis MW. Adaptive dynamics of infectious diseases: in pursuit of virulence management: Cambridge University Press; 2005. 8. Iwanami S, Kakizoe Y, Morita S, Miura T, Nakaoka S, Iwami S. A highly pathogenic simian/human immunodeficiency virus effectively produces infectious virions compared with a less pathogenic virus in cell culture. Theor Biol Med Model. 2017;14(1):9. doi: 10.1186/s12976-017-0055-8. PubMed PMID: 28431573; PubMed Central PMCID: PMCPMC5401468.

9. Iwami S, Holder BP, Beauchemin CA, Morita S, Tada T, Sato K, et al. Quantification system for the viral dynamics of a highly pathogenic simian/human immunodeficiency virus based on an in vitro experiment and a mathematical model. Retrovirology. 2012;9(1):18. Epub 2012/03/01. doi: 10.1186/1742-4690-9-18. PubMed PMID: 22364292; PubMed Central PMCID: PMCPMC3305505.

10. Kitagawa K, Nakaoka S, Asai Y, Watashi K, Iwami S. A PDE multiscale model of hepatitis $C$ virus infection can be transformed to a system of ODEs. J Theor Biol. 2018;448:80-5. Epub 2018/04/11. doi: 10.1016/j.jtbi.2018.04.006. PubMed PMID: 29634960.

11. Kitagawa K, Kuniya T, Nakaoka S, Asai Y, Watashi K, Iwami S. Mathematical Analysis of a Transformed ODE from a PDE Multiscale Model of Hepatitis C Virus Infection. Bull Math Biol. 2019;81(5):1427-41. Epub 2019/01/16. doi: 10.1007/s11538-018-00564-y. PubMed PMID: 30644067.

12. Iwami S, Takeuchi JS, Nakaoka S, Mammano F, Clavel F, Inaba H, et al. Cell-to-cell infection by HIV contributes over half of virus infection. Elife. 2015;4:e08150. Epub 2015/10/07. doi: 10.7554/eLife.08150. PubMed PMID:

370 26441404; PubMed Central PMCID: PMCPMC4592948. 
371 13. Ohashi H, Nishioka K, Nakajima S, Kim S, Suzuki R, Aizaki H, et al. The aryl hydrocarbon receptor-cytochrome P450 1A1 pathway controls lipid accumulation and enhances the permissiveness for hepatitis $C$ virus assembly. J Biol Chem.

374 2018;293(51):19559-71. Epub 2018/11/02. doi: 10.1074/jbc.RA118.005033. PubMed 375 PMID: 30381393; PubMed Central PMCID: PMCPMC6314116.

376 14. Nowak M, May RM. Virus dynamics: mathematical principles of immunology 377 and virology: mathematical principles of immunology and virology: Oxford University 378 Press, UK; 2000.

379 15. Soetaert K, Petzoldt T. Inverse Modelling, Sensitivity and Monte Carlo

380 Analysis in R Using Package FME. J Stat Softw. 2010;33(3):1-28. doi:

381 10.18637/jss.v033.io3. PubMed PMID: WOS:000275203400001. 16. R Core Team. R: A Language and Environment for Statistical Computing. R Foundation for Statistical Computing; 2019.

384 17. Haario H, Laine M, Mira A, Saksman E. DRAM: Efficient adaptive MCMC.

385 Stat Comput. 2006;16(4):339-54. doi: 10.1007/s11222-006-9438-0. PubMed PMID: 386 WOS:000241260600002.

387 18. Plummer M, Best N, Cowles K, Vines K. CODA: convergence diagnosis and output analysis for MCMC. R news. 2006;6(1):7-11. 
391

392

393

394

395

396

397

398

399

400

401

402

403

404

405

406

407

408

409

410

411

412

413

414

415

416

417

418

419

420

421

\section{ACKNOWLEDGMENTS}

Huh7.5.1 cells were kindly provided by Dr. Francis Chisari at The Scripps Research Institute. This study was supported in part by Grants-in-Aid for JSPS Research Fellow 19J12319 (to S. Iwanami), 19 J21395 (to K.K.), Scientific Research (KAKENHI) Scientific Research B 18 KT0018 (to S.I.), $18 \mathrm{H} 01139$ (to S.I.), $16 \mathrm{H} 04845$ (to S.I.), $17 \mathrm{H04085}$ (to K.W.), Scientific Research in Innovative Areas 19H04839 (to S.I.), 18H05103 (to S.I.); AMED CREST $19 \mathrm{gm} 1310002$ (to S.I.); AMED J-PRIDE $19 \mathrm{fm} 0208006 \mathrm{~s} 0103$ (to S.I.), 19fm0208014h0003 (to S.I.), $19 \mathrm{fm} 0208019 \mathrm{~h} 0103$ (to S.I.), 19fm0208019j0003 (to K.W.); AMED Research Program on HIVIAIDS 19fk0410023s0101 (to S.I.); Research Program on Emerging and Re-emerging Infectious Diseases 19fk0108050h0003 (to S.I.); Program for Basic and Clinical Research on Hepatitis 19fk0210036h0502 (to S.I.), 19fk0210036j0002 (to K.W.); Program on the Innovative Development and the Application of New Drugs for Hepatitis B 19fk0310114h0103 (to S.I.), 19fk0310114j0003 (to K.W.), 19fk0310101j1003 (to K.W.), 19fk0310103j0203 (to K.W.); JST MIRAI (to S.I. and K.W.); JST CREST (to S.I. and K.W.); Mitsui Life Social Welfare Foundation (to S.I. and K.W.); Shin-Nihon of Advanced Medical Research (to S.I.); Suzuken Memorial Foundation (to S.I.); Life Science Foundation of Japan (to S.I.); SECOM Science and Technology Foundation (to S.I.); The Japan Prize Foundation (to S.I.); Toyota Physical and Chemical Research Institute (to S.I.); The Yasuda Medical Foundation (to K.W.); Smoking Research Foundation (to K.W.); Takeda Science Foundation (to K.W.).

\section{AUTHOR CONTRIBUTIONS}

$\mathrm{OD}, \mathrm{S}$ Iwami and $\mathrm{KW}$ designed the research. $\mathrm{HO}, \mathrm{KN}$, and $\mathrm{KW}$ conducted the experiments. S Iwanami, KK, YA, $\mathrm{HI}$ and S Iwami carried out the computational analysis. OD, S Iwami and $\mathrm{KW}$ supervised the project. All authors contributed to writing the manuscript.

\section{COMPETING FINANCIAL INTERESTS}

The authors declare that they have no competing interests. 


\section{FIGURE LEGENDS}

423 Figure 1 | Schematic representation of multiscale HCV infection and mathematical model: (A) Schematic representation of intracellular HCV life cycle and trade-off between viral replication and release of intracellular viral RNA. Viral RNA in cells is translated to produce structural (S) and non-structural (NS) proteins. Viral RNA is either amplified through the functions of NS proteins through replication, or is assembled with $S$ proteins and released as a progeny virus. If the balance between viral replication and release leans toward replication, intracellular viral RNAs will accumulate. In contrast, high rates of intracellular RNA release will create opportunities for transmission to new cells but will deplete viral RNA in the cell. (B) Modeling the intracellular virus life cycle. Intracellular viral RNA either replicates inside the cell at rate $k$, is degraded at rate $\mu$, or assembles with viral proteins to be released within $\mathrm{HCV}$ virions at rate

p. (C) Multiscale modeling of intracellular replication and intercellular infection. Target cells are infected by infectious viruses at rate $\beta$.

Figure 2 | Dynamics of HCV JFH-1 and Jc1-n infection in cell culture. (A) Fitting of the mathematical model to the experimental data of HCV JFH-1 and Jc1-n infection in cell culture. Three different numbers of Huh-7 cells infected with either HCV JFH-1 or Jc1-n 1 day after inoculation were seeded (Experiment A: 1000, Experiment B: 2000, and Experiment C: 4000 cells per well) and chased to detect the following values at days $0,1,2,3$, and 4 post-seeding ( $\log _{10}$ scale): numbers of uninfected and infected cells, amount of intracellular and extracellular viral RNA (copies/well), and extracellular viral infectivity (ffu/well) (orange circle: JFH-1, green triangle: Jc1-n). The shadowed regions correspond to $95 \%$ posterior intervals and the solid curves give the best-fit solution (mean) for Eqs. (2-6) to the time-course dataset. All data for each strain were fitted simultaneously. (B) Dynamics of the distributions of intracellular viral RNA according to infection age, $a$. The distributions were calculated using the original multiscale PDE model (Eqs. (S2-6) in Supplementary Note 1) using means of estimated parameters for HCV JFH-1 and Jc1-n. The colored bars represent the amount of intracellular viral RNA. (C) Difference in the distributions of intracellular viral RNA in total infected cells of infection age, $a$, between HCV JFH-1 and Jc1-n.

454 The colored bar shows the difference in the amount of intracellular viral RNA (green: intracellular viral RNA during $\mathrm{Jc} 1-\mathrm{n}$ infection is more abundant than during $\mathrm{JFH}-1$ 
456 infection, yellow-red-brown: intracellular viral RNA is more abundant for JFH-1 than

457 for Jc1-n, gray: no new infection occurs due to depletion of target cells).

Figure 3 | Characterization of viral dynamics of HCV JFH-1 and Jc1-n. The distributions of the rate constant for infection, $\beta_{\theta}$, the release rate of intracellular viral RNA, $\rho$, the degradation rate of intracellular viral RNA, $\mu$, the converted fraction of infectious viral RNA, $f_{\theta}$, and the replication rate of intracellular viral RNA, $k$, inferred by MCMC computations are shown in (A), (B), (C), (D) and (E), respectively, for HCV JFH-1 (orange) and Jc1-n (green). Parameters $\beta_{\theta}, \rho$ and $\mu$ for Jc1-n were significantly larger than for JFH-1, while there was no significant difference in $f_{\theta}$ between the two strains as assessed by repeated bootstrap t-test. JFH-1 and Jc1-n stains had identical viral RNA replication rates. The distributions of accumulation rates of intracellular viral RNA, $k-\mu-\rho$, and the Malthusian parameter, $M$, calculated from all accepted MCMC parameter estimates are shown in $(\mathbf{F})$ and $(\mathbf{G})$, respectively, for HCV JFH-1 (orange) and Jc1-n (green). These indices were significantly larger for $\mathrm{JFH}-1$ than for $\mathrm{Jc} 1-\mathrm{n}$ as assessed by the repeated bootstrap t-test.

Figure 4 | Different strategies adopted by JFH-1 and Jc1-n for viral proliferation.

(A) Schematic representation of the fate of replicated intracellular viral RNA. Viral RNA is used either for driving RNA replication in cells, for producing progeny viruses HCV JFH-1 and Jc1-n viral RNA that remains inside cells, is released outside cells, and is degraded. (C) Change in the Malthusian parameter with various release rates of intracellular viral RNA. The orange and green curves show Malthusian parameters calculated using 100 parameter sets sampled from MCMC parameter estimates as functions of $\rho$ for JFH-1 and Jc1-n, respectively. The gray vertical lines are the corresponding release rates estimated from the actual experimental data. (D) Change in the cumulative number of newly infected cells with the various release rates. The orange and green curves represent the cumulative number of newly infected cells until 2, 4, 6, 8, and 10 days post-infection calculated using the means of estimated parameters as function of $\rho$ for JFH-1 and Jc1-n, respectively. The gray vertical line represents the mean release rate estimated from the experimental data. 
Table 1. Parameter values estimated from the cell culture infection experiment.

\begin{tabular}{|c|c|c|c|c|c|c|}
\hline \multirow{2}{*}{ Parameter Name } & \multirow{2}{*}{ Symbol } & \multirow{2}{*}{ Unit } & \multicolumn{2}{|c|}{ HCV JFH-1 } & \multicolumn{2}{|c|}{ HCV Jc1 } \\
\hline & & & Value & $95 \% \mathrm{Cl}$ & Value & $95 \% \mathrm{Cl}$ \\
\hline \multicolumn{7}{|l|}{ Fitted parameters from separate experiments } \\
\hline Rate of virion infectivity loss & $r$ & day $^{-1}$ & 1.60 & - & 2.40 & - \\
\hline Degradation rate of extracellular viral RNA & $c_{R N A}$ & day $^{-1}$ & 0.08 & - & 0.24 & - \\
\hline Clearance rate of extracellular viral RNA & $c_{W}$ & day $^{-1}$ & 1.18 & - & 1.82 & - \\
\hline Reduction rate of intracellular viral RNA & $\mu+\rho$ & day $^{-1}$ & 0.80 & - & 0.89 & - \\
\hline \multicolumn{7}{|c|}{ Estimated parameters from in vitro total cell growth data } \\
\hline Proliferation rate of Huh-7 cells & $g$ & day $^{-1}$ & 0.67 & - & 0.67 & - \\
\hline Carrying capacity of Huh- 7 cells & $K$ & cells & $4.12 \times 10^{4}$ & - & $3.75 \times 10^{4}$ & - \\
\hline \multicolumn{7}{|c|}{ Parameters obtained from simultaneous fitting of full in vitro dataset } \\
\hline Rate constant for infections & $\beta_{\theta}$ & $(\mathrm{ffu} / \text { well } \cdot \text { day })^{-1}$ & $1.29 \times 10^{-4}$ & $0.81-1.92 \times 10^{-4}$ & $2.21 \times 10^{-4}$ & $1.69-2.77 \times 10^{-4}$ \\
\hline Replication rate of intracellular viral RNA & $k$ & day $^{-1}$ & 1.91 & $1.84-1.98$ & 1.91 & $1.84-1.98$ \\
\hline Release rate of intracellular viral RNA & $\rho$ & day $^{-1}$ & $2.43 \times 10^{-2}$ & $1.87-3.11 \times 10^{-2}$ & $6.25 \times 10^{-2}$ & $4.62-8.44 \times 10^{-2}$ \\
\hline Degradation rate of intracellular viral RNA & $\mu$ & day $^{-1}$ & 0.78 & $0.77-0.78$ & 0.83 & $0.80-0.84$ \\
\hline Converted fraction of infectious viral RNA & $f_{\theta}$ & RNA copies $\cdot \mathrm{ffu}^{-1}$ & $1.21 \times 10^{-3}$ & $0.86-1.67 \times 10^{-3}$ & $1.13 \times 10^{-3}$ & $0.83-1.49 \times 10^{-3}$ \\
\hline
\end{tabular}




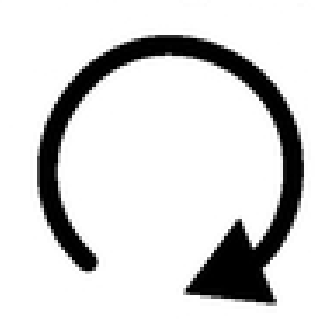

Intracellular W viral RNA W W Wheleased: $\rho / k$

bioRxiv preprint doi: https://doi.org/10.1101/82171 was not certified by peer review) is the author/fun

version posted October 28, 2019. The copyright holder for this preprint (which has granted bioRxiv a license to display the preprint in perpetuity. It is made availab

\section{Degraded: $\mu / k$}

\section{JFH-1}

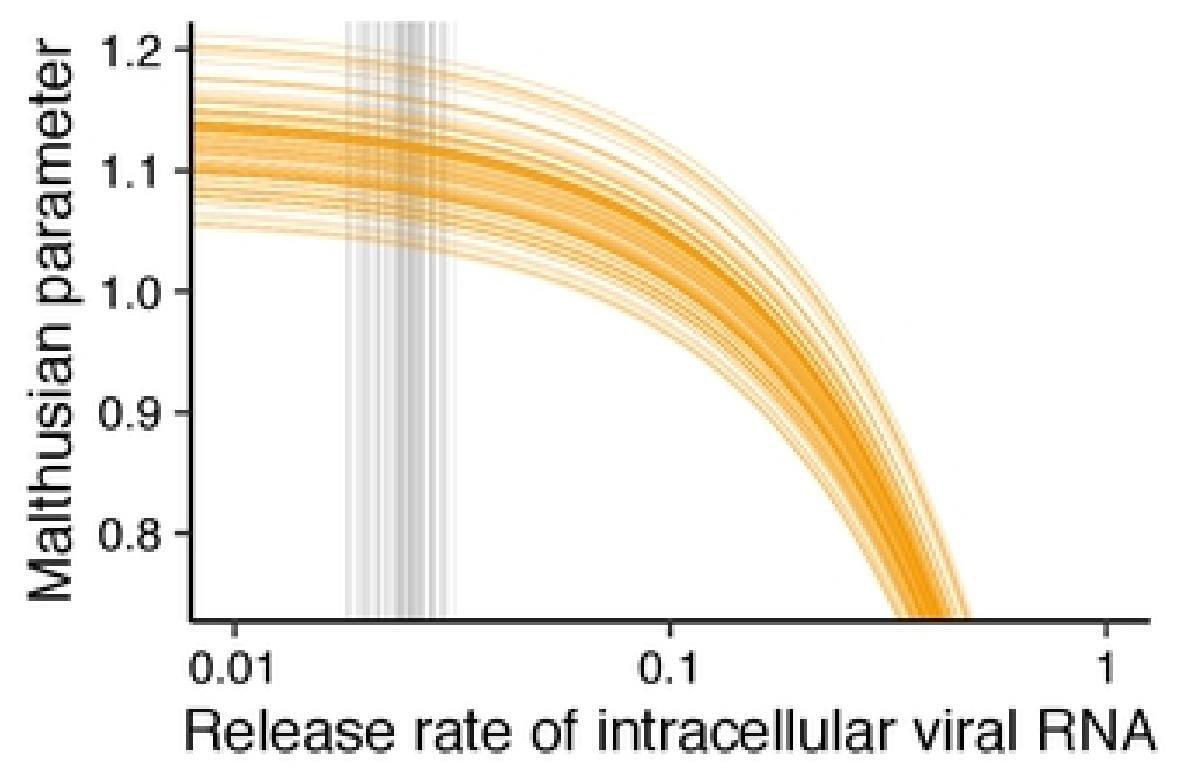

Jc1-n

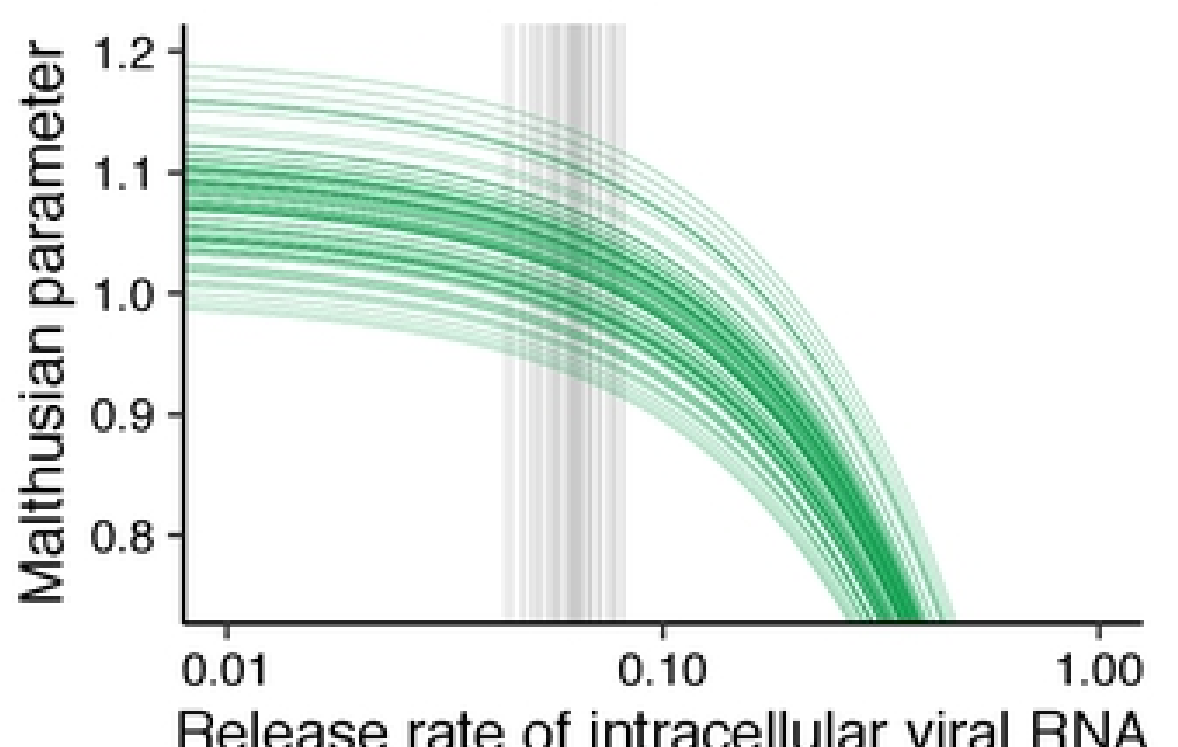

D
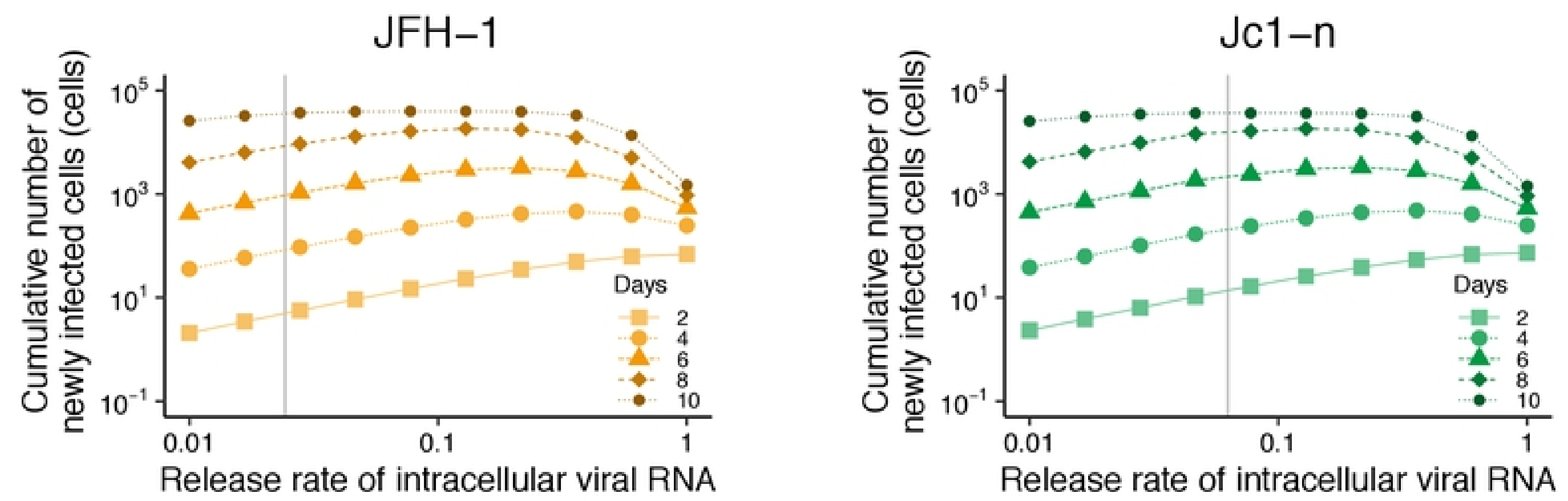

Fig4 


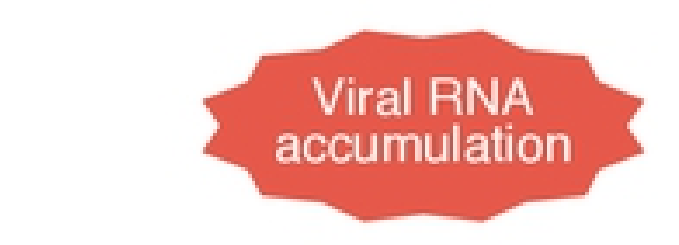

Replication 1

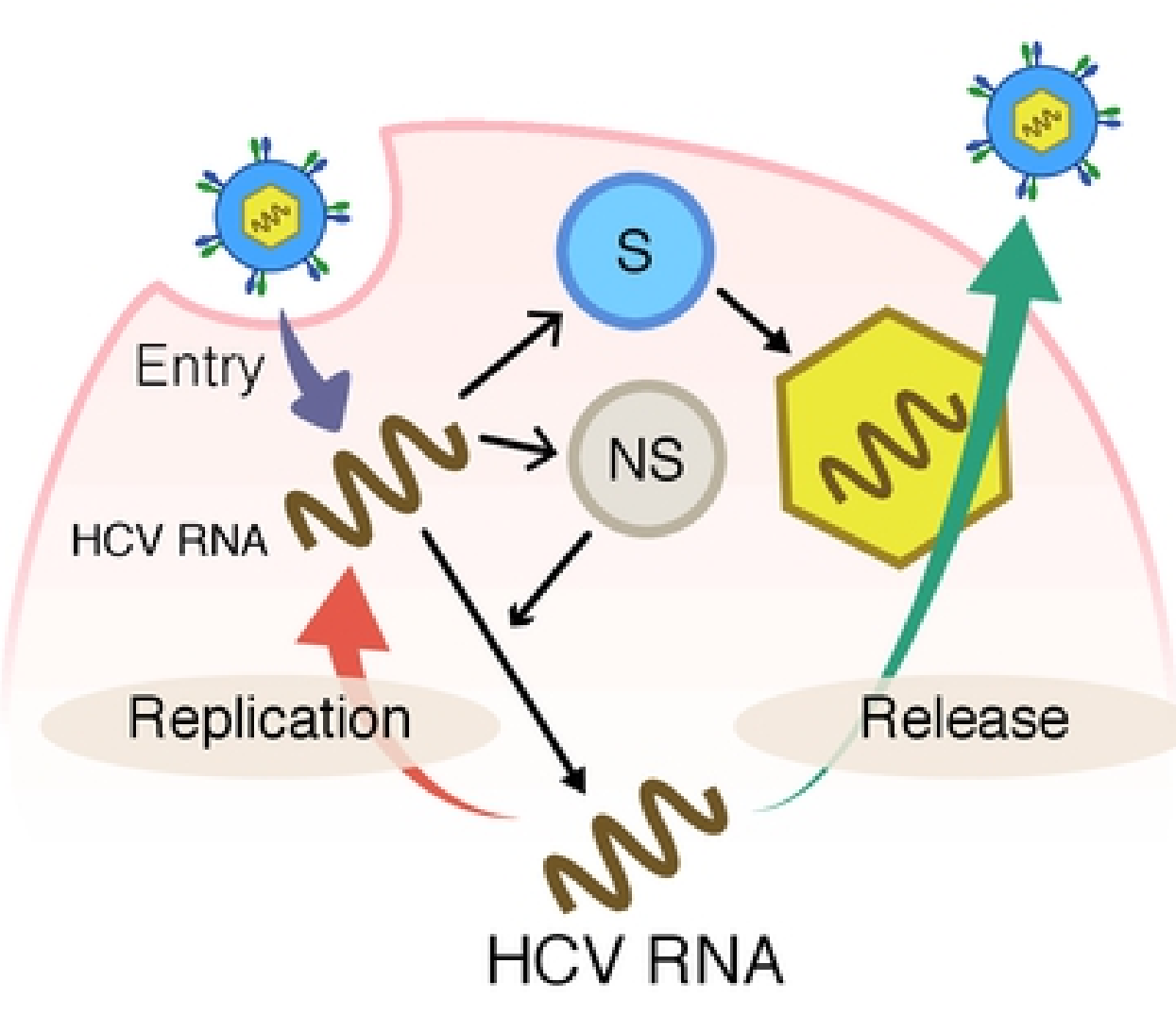

C

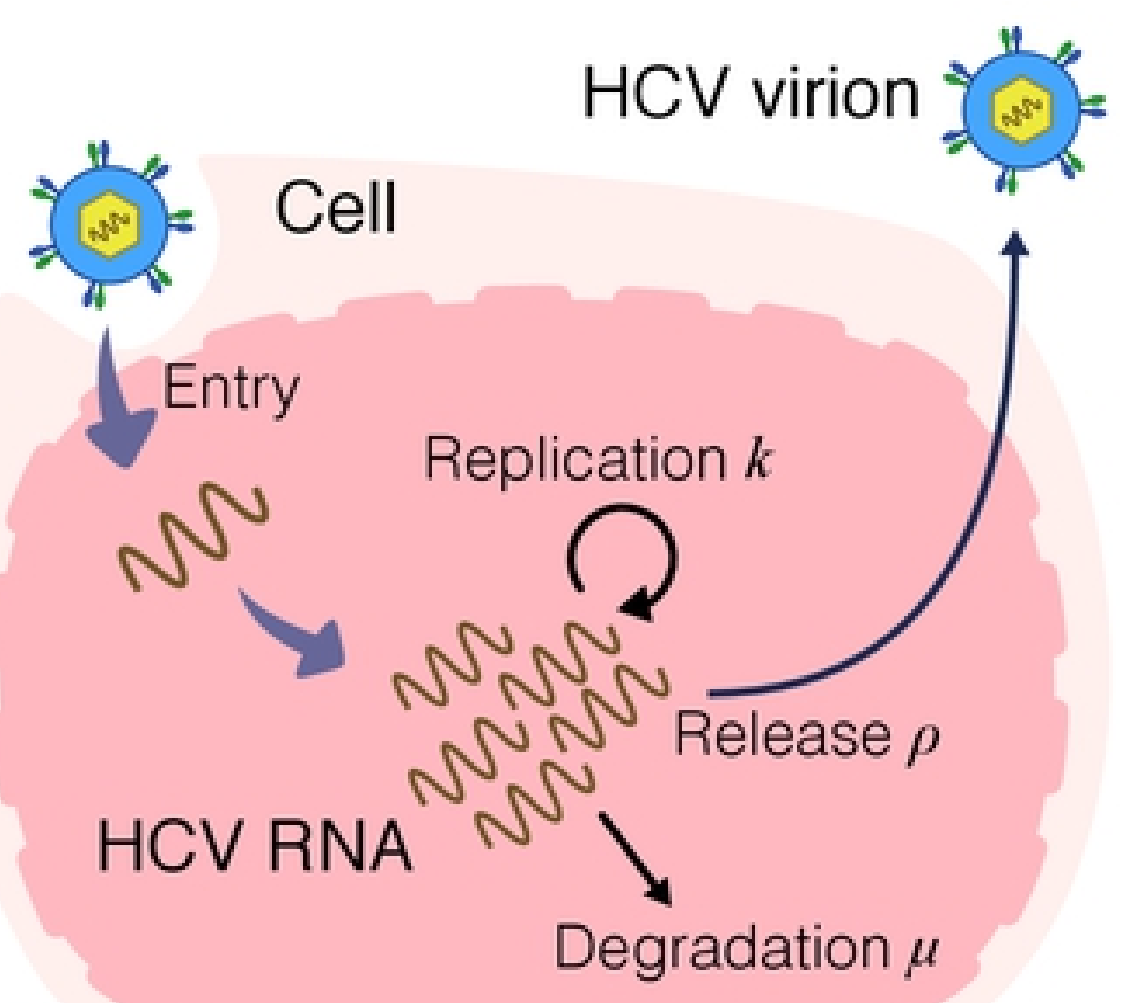

Target cells

Infected cells

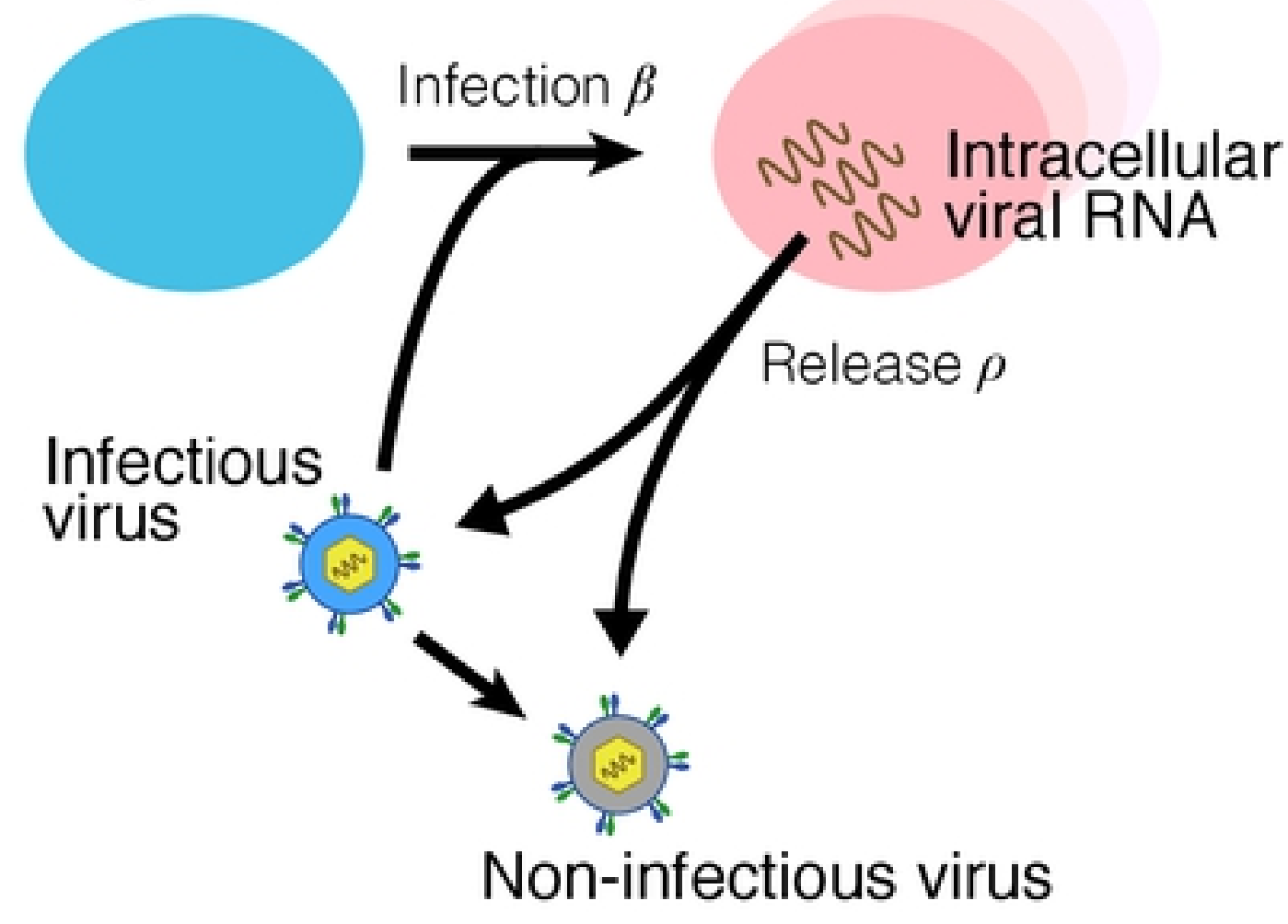

Fig1 
A

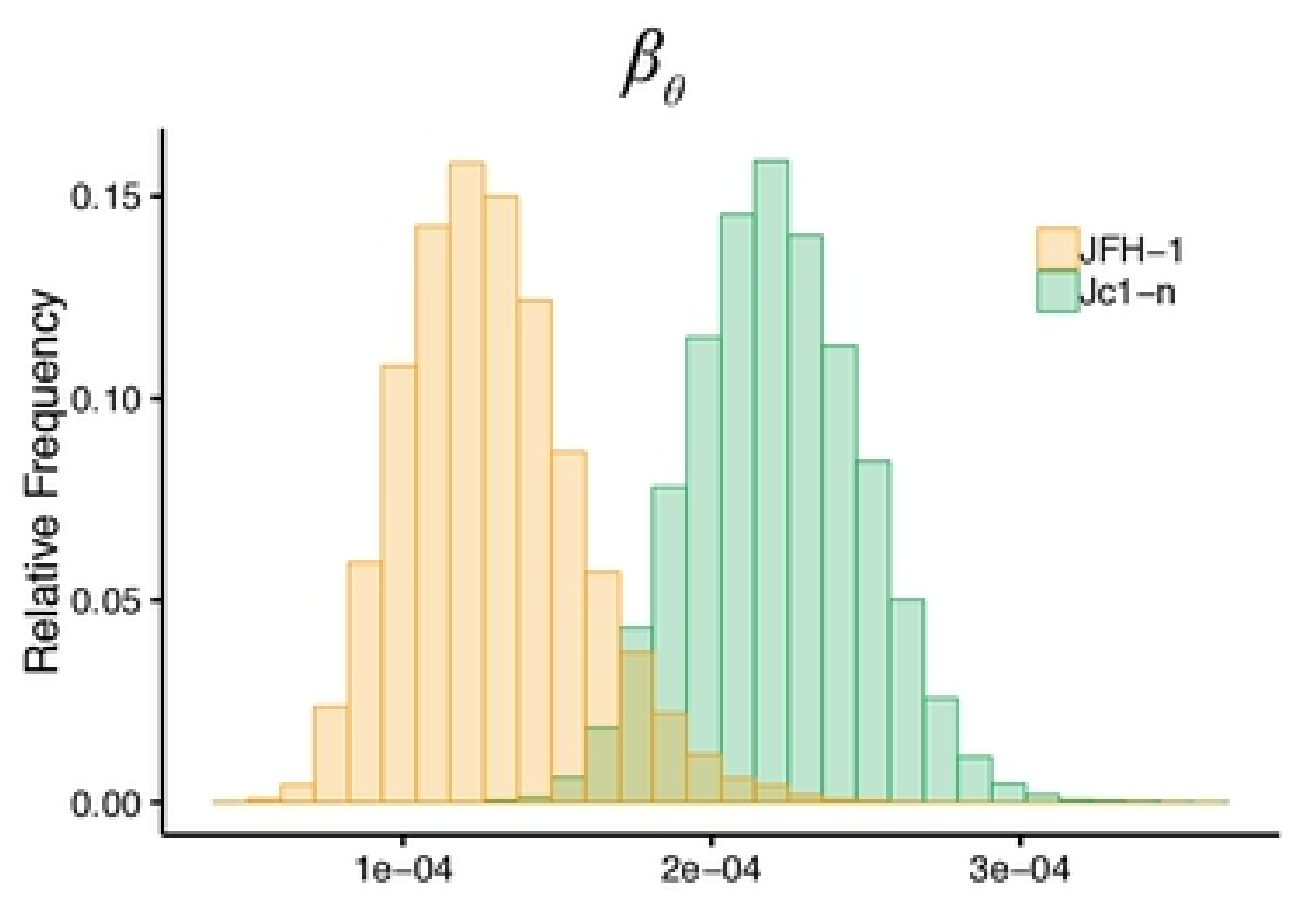

$f_{\theta}$

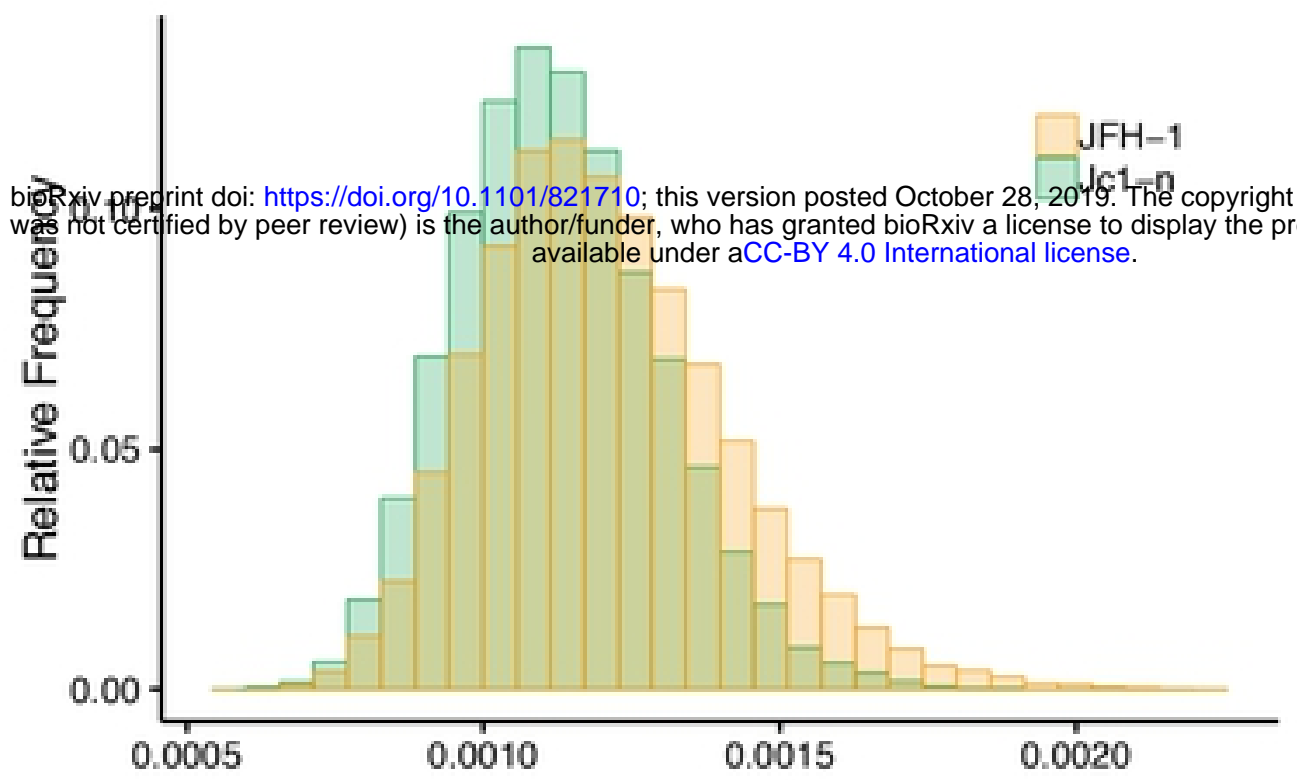

E

$k$

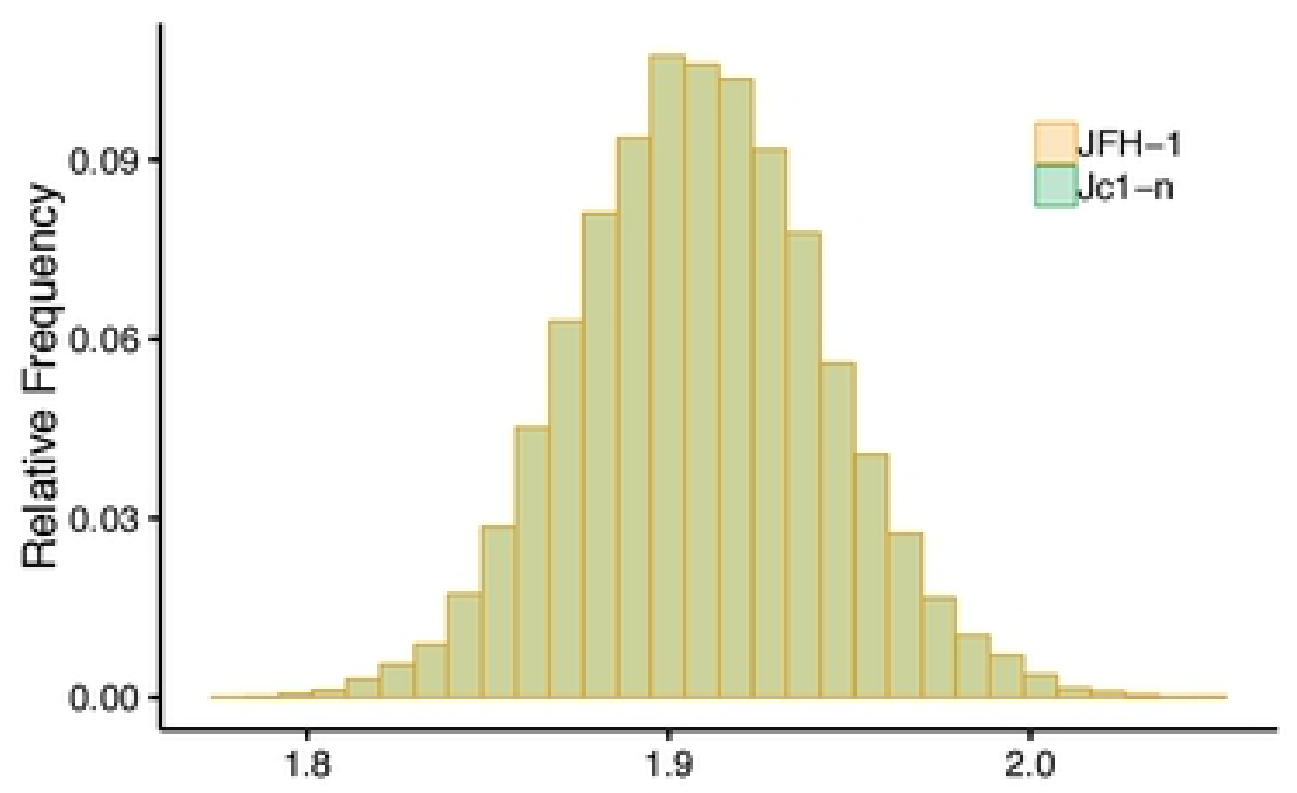

G

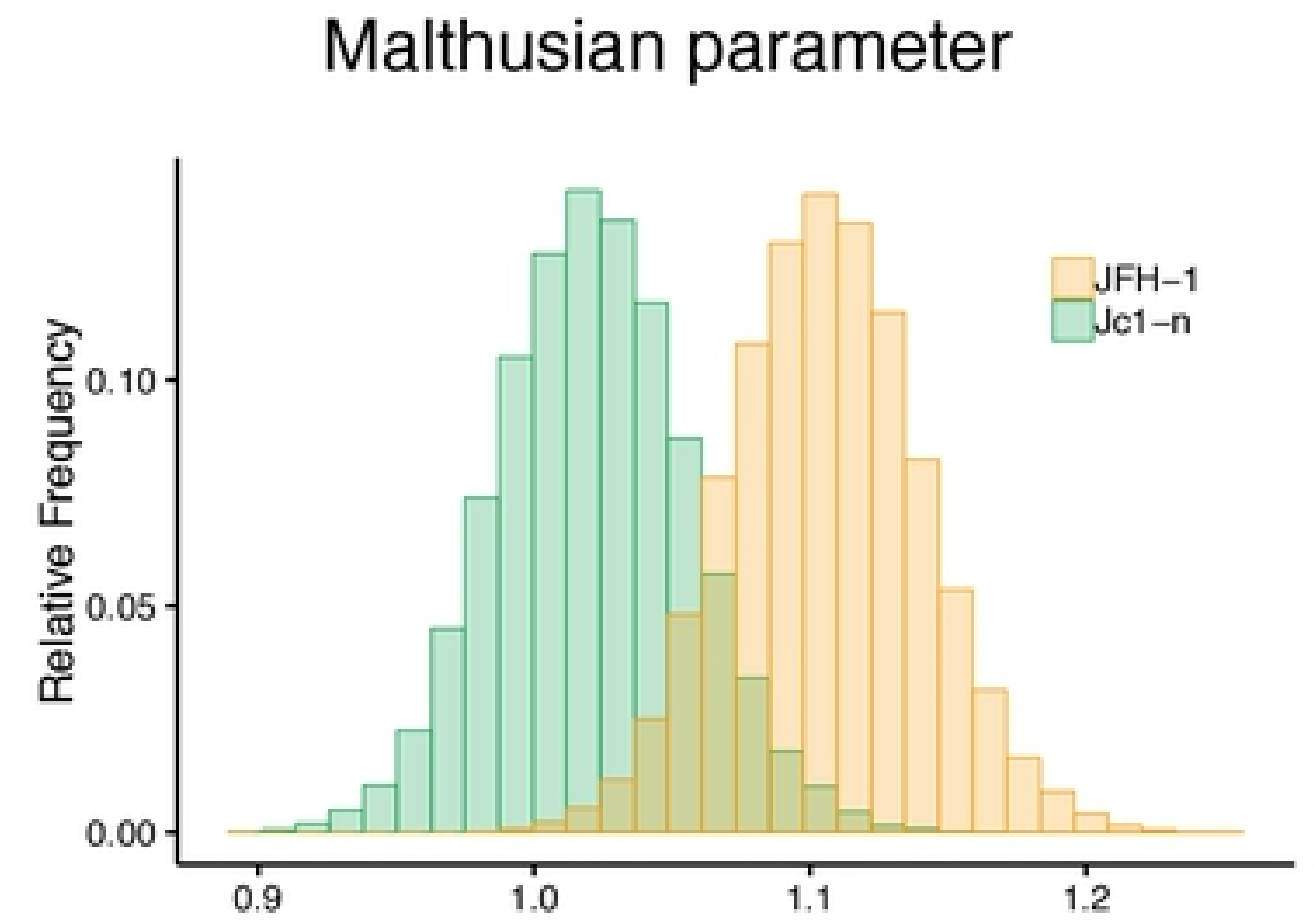

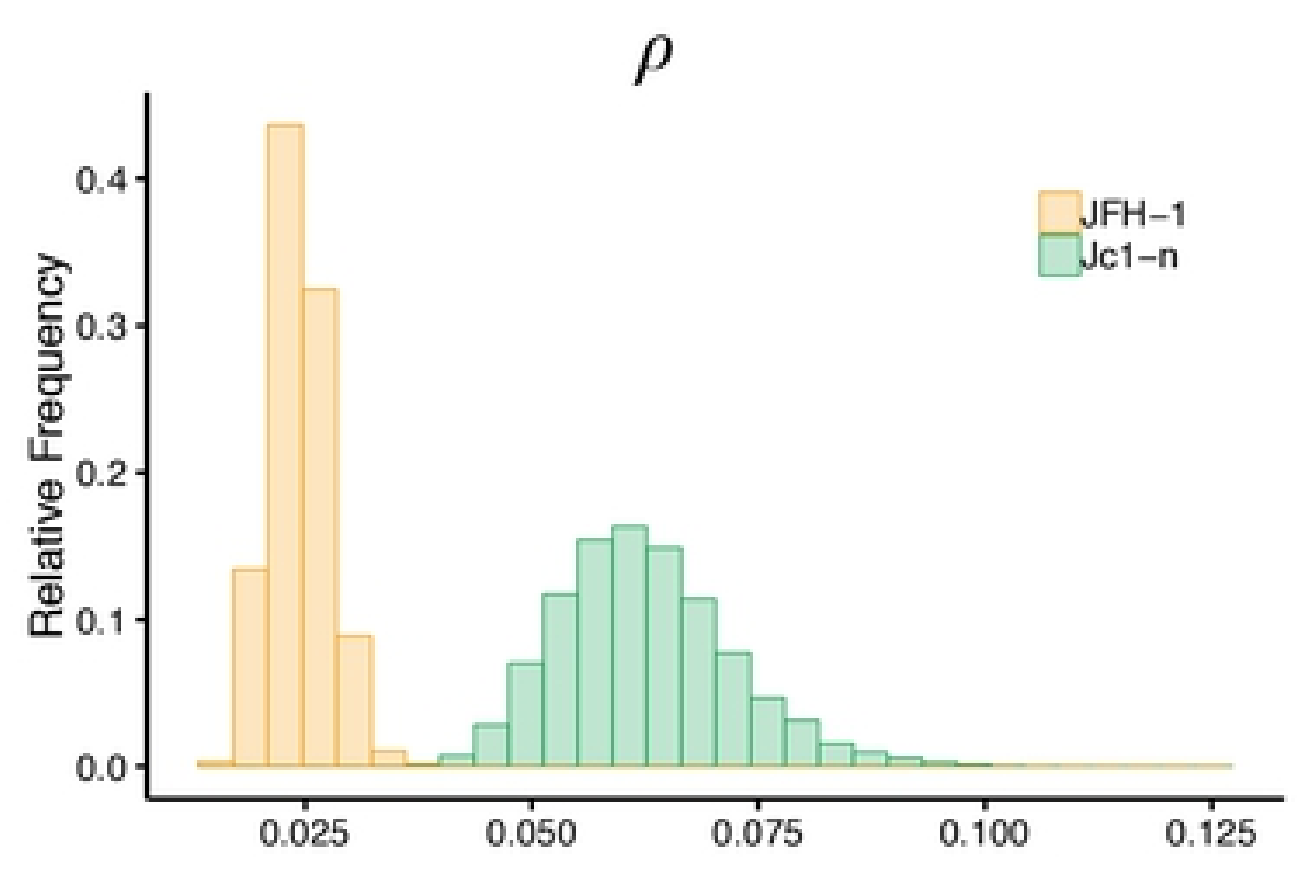

D
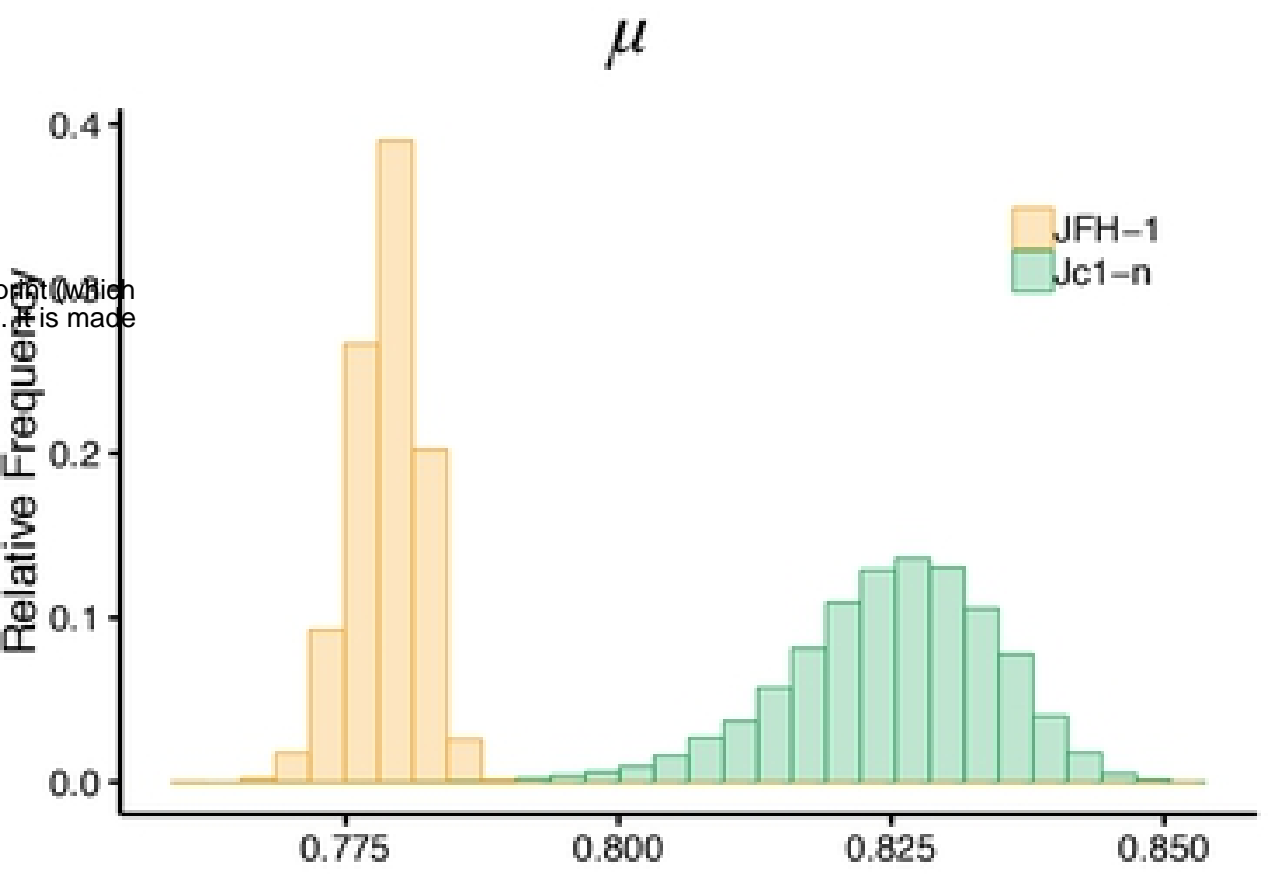

F Accumulation rate of intracellular viral RNA

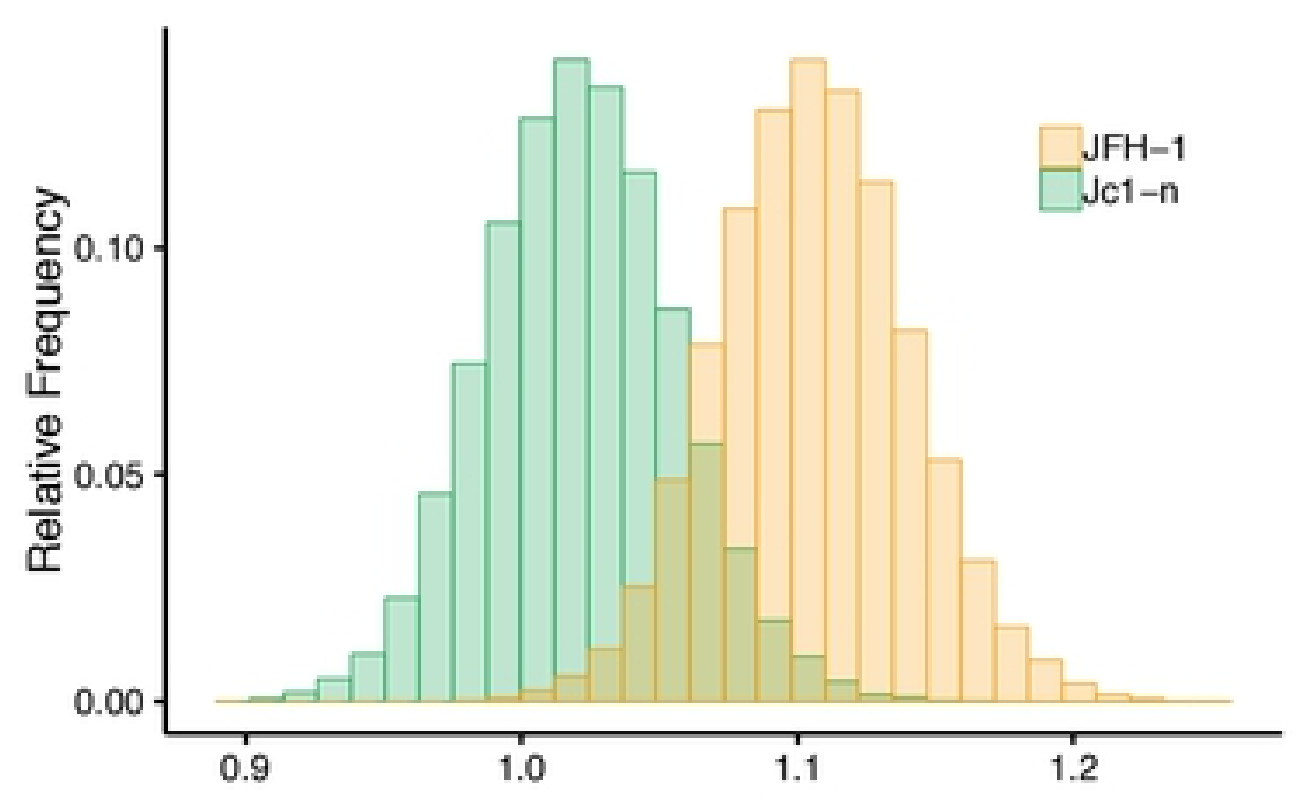

Fig3 
Target cells Infected cells $\begin{gathered}\text { Intracellular } \\ \text { viral RNA }\end{gathered}$
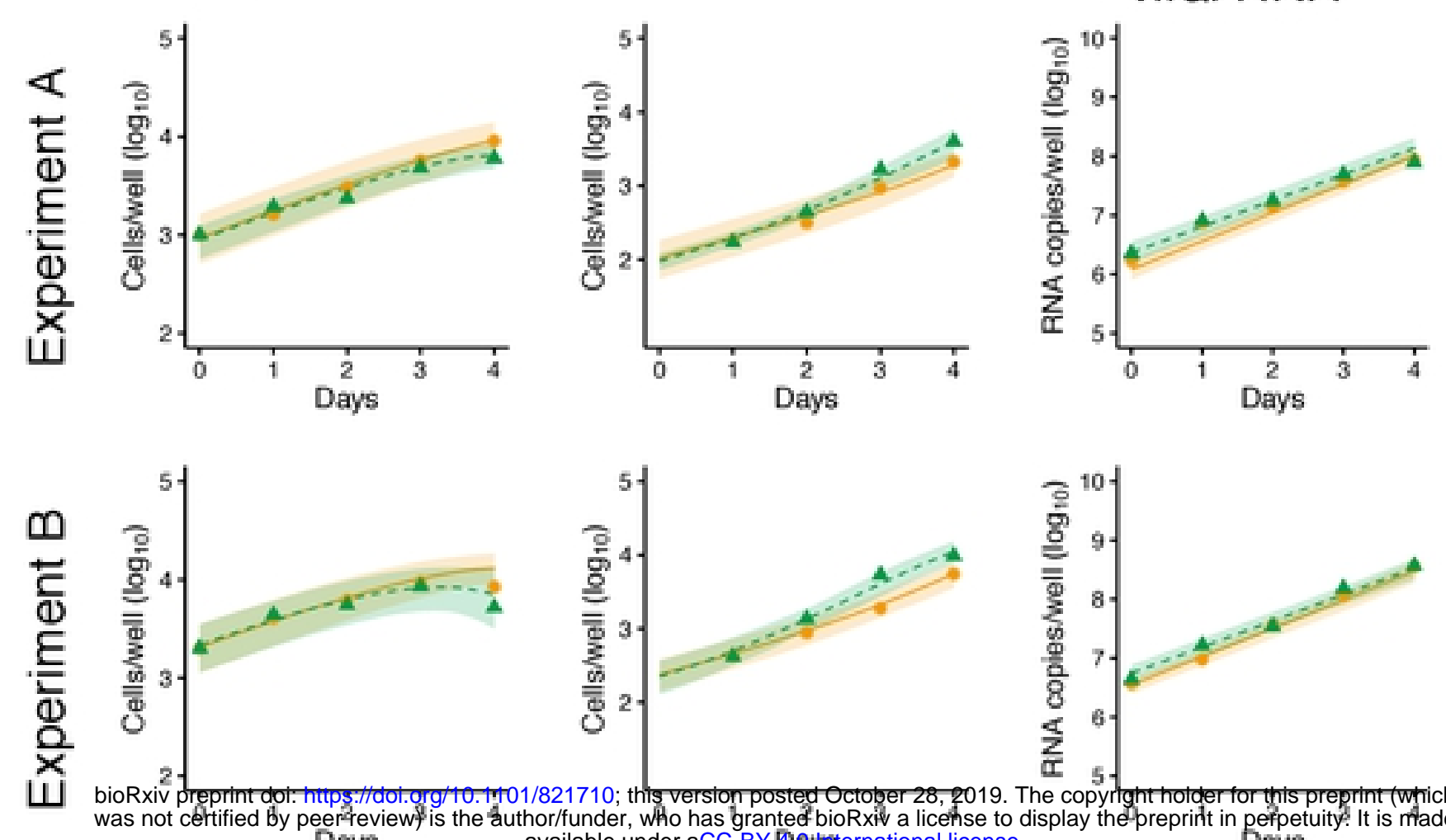
available under aCC-BY W.aysioternational license.
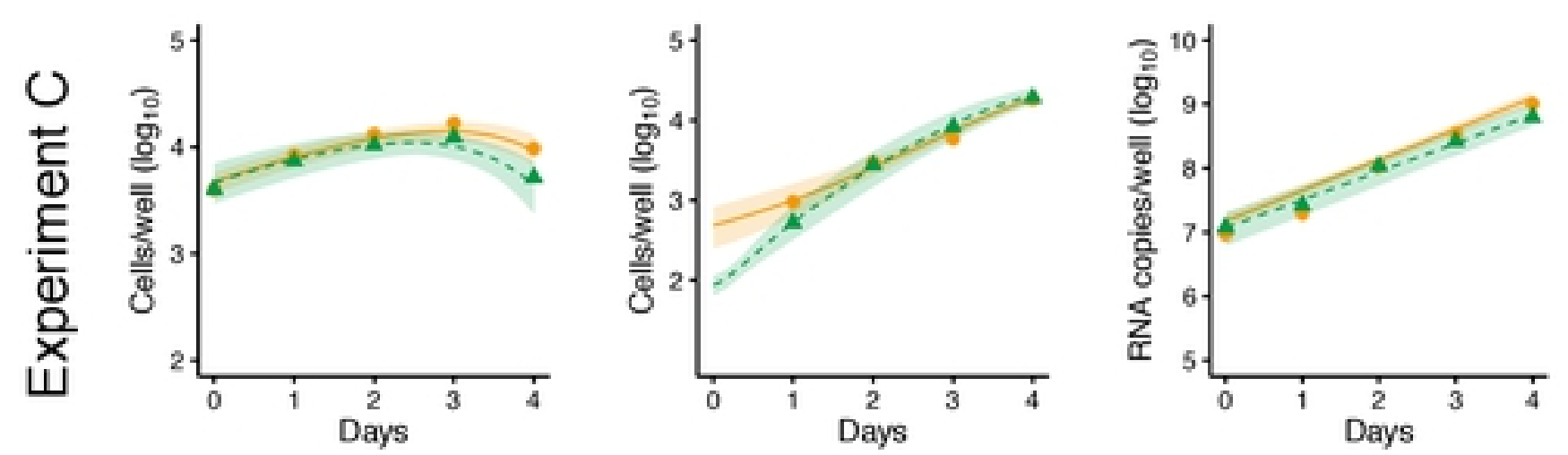

95\% credible intreval and Best-fit solution

Experimental data

$\mathrm{JFH}-1 \quad \Delta \mathrm{Jc} 1-\mathrm{n}$

JFH-1 Average
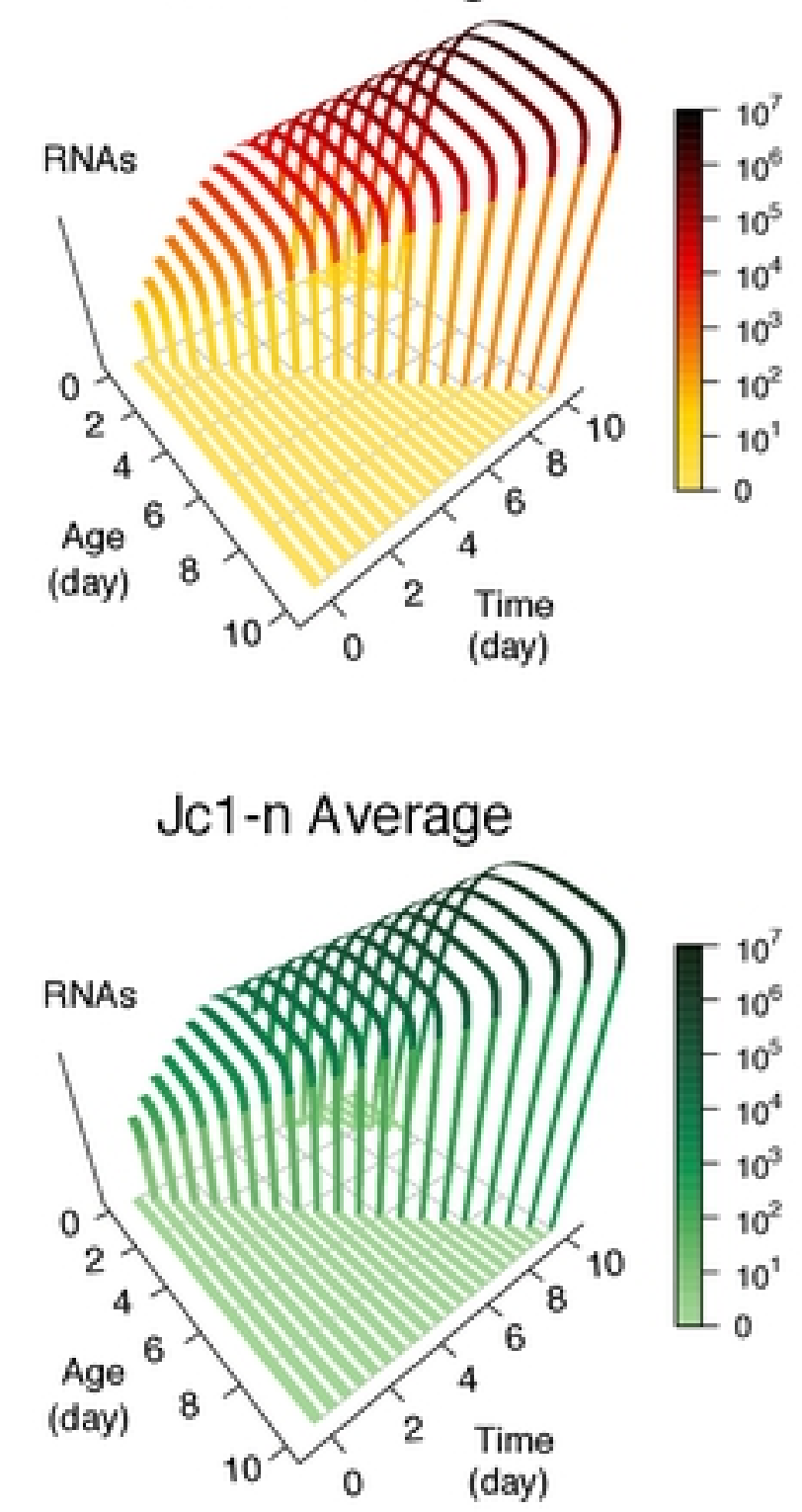

B

Figure
C

JFH-1 .... Jc1-n
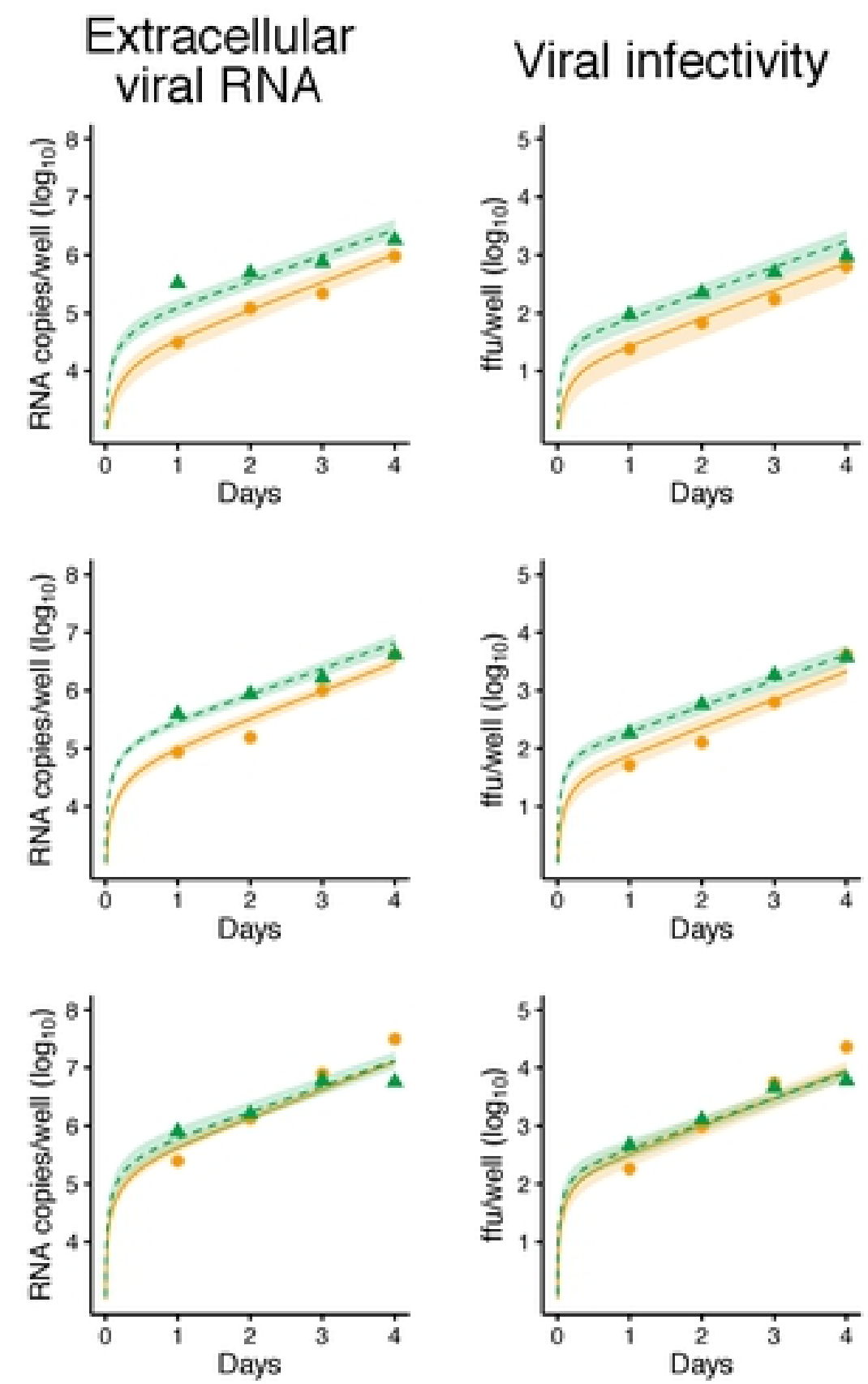

Difference between JFH-1 and Jc1-n

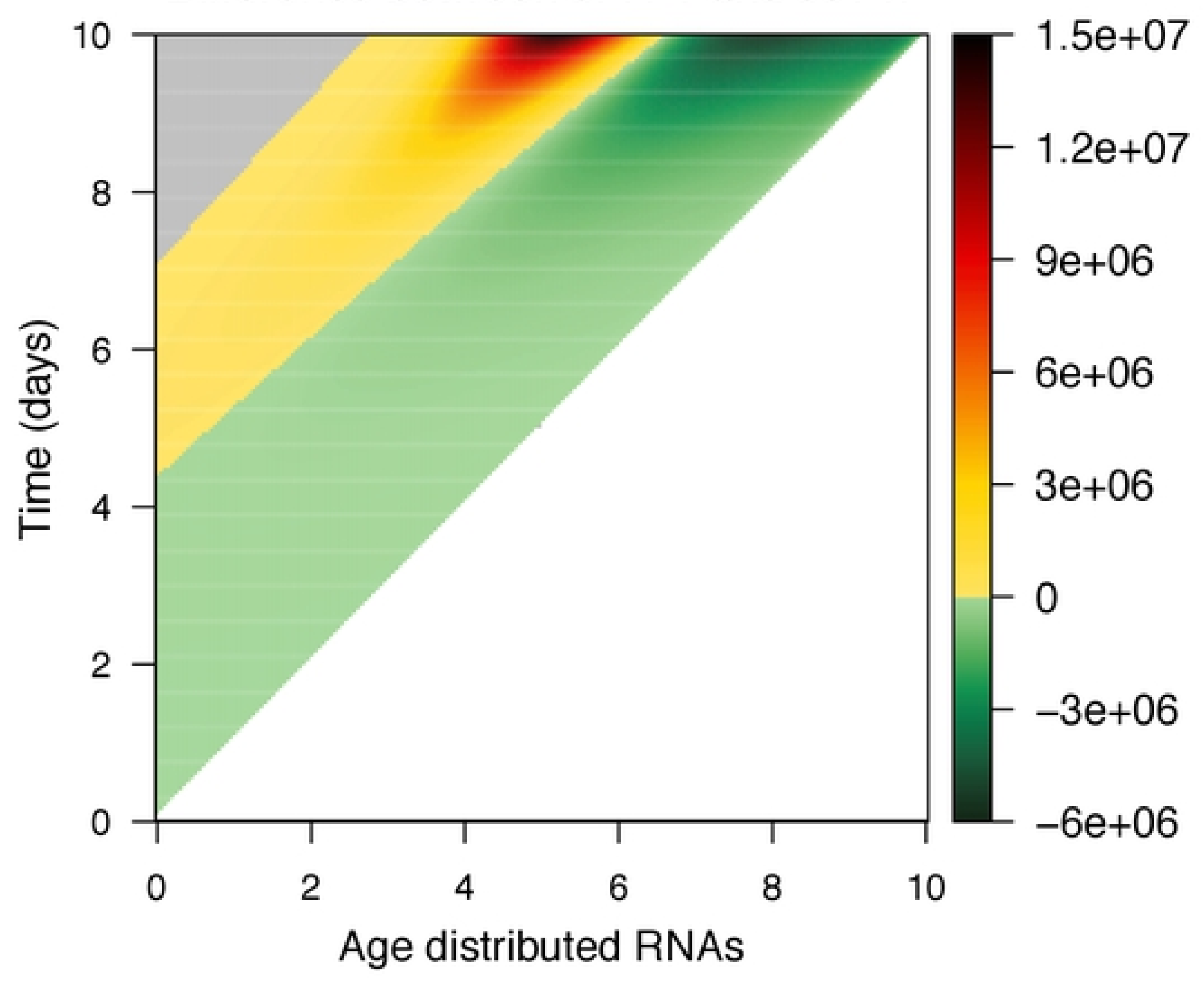

